\title{
EI corte de rocas ornamentales con discos diamantados: influencia de las características petrográficas de la roca
}

\author{
A. RODRÍGUEZ-REY, L. M. SUÁREZ DEL RÍO, L. CALLEJA y V. G. RUIZ DE ARGANDOÑA
} Dpto. de Geología. Área de Petrología y Geoquímica. Univ. de Oviedo

Fecha de recepción: 20-X-97

Fecha de aceptación: 11-II-98

ESPAÑA

\begin{abstract}
RESUMEN
Este trabajo es continuación de otro ya publicado en un número anterior de esta revista, en el que se revisaban algunos trabajos cientificos sobre la influencia de los factores propios del sistema de corte (sierra, parámetros de corte, características del disco, etc.) en el rendimiento del proceso de corte de rocas ornamentales con discos diamantados. En esta segunda parte, se consideran las características propias de la roca (mineralogia, textura y espacios vacios) que afectan a dicho rendimiento. También se hace una revisión de algunas clasificaciones de rocas (en función de su cortabilidad) propuestas por diversos autores $e$ Instituciones.
\end{abstract}

\section{SUMMARY}

This paper follows another one published in the preceding number of this same journal, that reviewed some scientific papers on the influence of the factors of the cutting equipments (machinary, cutting characteristics, disks, etc) on the efficiency of the cutting process of ornamental rocks with diamond disks. In this second part, the inner rock characteristics (mineralogy, texture and voids) affecting the cutting performance are considered. Some rock classifications based on their cuttability proposed by some authors and Institutions are also reviewed.

\section{INTRODUCCIÓN}

En un trabajo anterior (1) se comentó el importante cambio que la incorporación del diamante a los procesos de extracción y elaboración de rocas ornamentales ha supuesto en la tecnología de la Piedra Natural. De aquí la importancia de los estudios acerca de la capacidad de corte de rocas con útiles diamantados, especialmente discos.

Dado que en el proceso de corte se producen dos fenómenos recíprocos (el útil diamantado corta a la roca que, a su vez, desgasta al útil), el estudio puede enfocarse desde dos perspectivas distintas y, a su vez, complementarias. En e] mencionado artículo se exponian algunas consideraciones respecto al papel desempeñado por las características del útil de corte, condiciones de corte, etc. En esta segunda parte, se estudia la influencia de las características petrográficas de las rocas en el proceso de corte con discos diamantados
Las características propias de la roca ejercen una influencia en el proceso de corte, tanto a una "macroescala" (donde la influencia de las discontinuidades -diaclasas, fracturas, planos de estratificación, etc.- así como de los esfuerzos tectónicos en el afloramiento, son muy importantes) como a una "microescala" (donde el corte se realiza a escala de "roca matriz", libre de discontinuidades macroscópicas, y donde son las características petrográficas de la roca -mineralogía, textura y espacios vacíos- las que ejercen su influencia).

Howarth et al. (2), que reconocen estas dos escalas, señalan que es a escala "micro" donde pueden investigarse los mecanismos fundamentales de corte, que se ven afectados por la mineralogía, textura y microestructura de la roca. Esta escala "micro" es la que debe ser considera. da en el procesado de rocas ornamentales, cuyos bloques son "roca matriz" exenta de discontinuidades que los haría inservibles como tales. 
Previamente consideramos interesante referirnos brevemente a los mecanismos mediante los cuales se produce el corte de la roca.

\section{MECANISMOS DE CORTE DE ROCAS}

Los mecanismos que actúan durante el corte de materiales rocosos han sido estudiados mediante ensayos de indentación, consistentes en aplicar cargas compresivas con indentadores de formas diversas sobre la superficie de la roca.

El proceso de corte se produce por la trituración y deformación inelástica de la roca justo por debajo del indentador (en el corte con disco, el indentador sería el cristal de diamante) seguido de la formación de un "cráter" debido al crecimiento de fisuras laterales desde la punta del indentador hasta la superficie ("surface chipping"). Swain y Lawn(3) describen el proceso de fisuración de una roca durante un ensayo de microindentación en un material frágil (como las rocas). En la figura 1 se representa esquemáticamente este proceso: cuando se aplica la carga, la roca debajo del indentador se deformará inelásticamente, produciendo una zona de trituración y la nucleación de algunas fisuras o craks (Figura 1 a); al aumentar la carga compresiva, crece una o varias fisuras medias (M) con forma circular en la dirección del plano de aplicación de carga (Fig. 1 b). Simultáneamente se forman unas fisuras dispuestas en forma de cono invertido (denominadas fisuras en cono, C) que terminan en la zona de trituración (Fig. 1 c). Durante la descarga puede disminuir la anchura de las fisuras creadas (se cierran ligeramente, Fig. $1 \mathrm{~d}$ ) y comienzan a crecer a partir de la zona de trituración unas fisuras laterales (L, Fig. 1 e) que llegan hasta la superficie (Fig. $1 \mathrm{f}$ ).
Si el indentador se desplaza (como es en el caso del cristal de diamante en el disco) se produce un surco cuyas características van a depender de la morfología del diamante indentador, de la carga compresiva aplicada, de la velocidad transversal y de las propiedades mecánicas (dureza, características elásticas, resistencia a la compresión, etc.) del material a cortar (4).

McGowan y Branninger (5) resumen los mecanismos de corte en tres etapas: a) Rayado; b) Fracturación creada por fuerzas compresivas; y c) Creación de fisuras resultantes del alivio de dichas fuerzas compresivas. Estas etapas coinciden con las descritas por Swain y Lawn (3).

El tamaño de las partículas arrancadas durante el aserrado de una roca puede darnos información respecto al desgaste (y, por tanto, a la vida útil) del disco de corte: a mayor tamaño de las partículas, mayor desgaste del $\operatorname{disco}(6)$.

El tamaño de estas partículas depende de cinco factores clave en el proceso de aserrado: diámetro del disco, profundidad del corte, velocidad periférica del disco, velocidad transversal y número de cristales activos de diamante en la superficie del disco, aunque los tres últimos son los más importantes. El tamaño de las partículas aumentaría al aumentar la velocidad transversal y disminuir la velocidad periférica del disco y la concentración de diamantes en los segmentos del dis$\operatorname{co}(7)$.

Es decir, para cortar un material determinado (manteniendo el mismo régimen de corte) se puede aumentar la vida del disco utilizando una velocidad transversal lenta y aumentando la profundidad de corte, así como utilizando

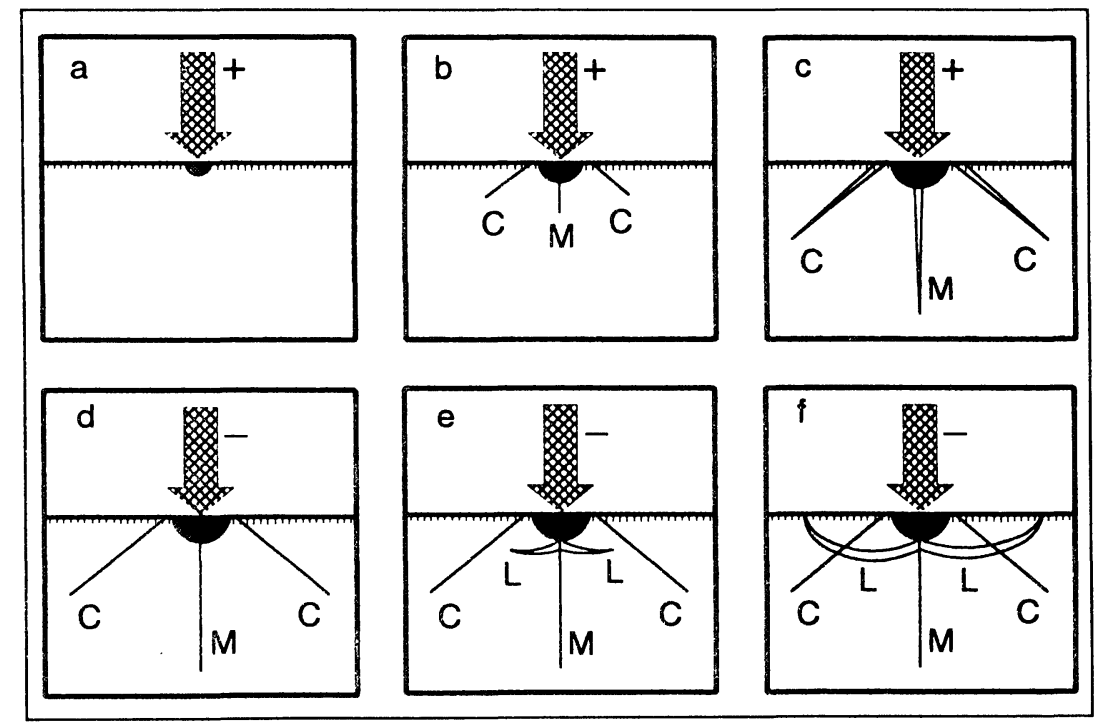

Fig. 1.- Proceso de fisuración durante un ensayo de microindentación en un material frágil (3). 
una velocidad periférica óptima del disco, como se ha visto anteriormente (1). Esto estaría relacionado con la fuerza que actúa sobre las partículas de diamante: cuanto mayores sean los esfuerzos sobre los diamantes, mayor va a ser el tamaño de las partículas arrancadas al material y menor va a ser la vida útil del disco.

\section{INFLUENCIA DE LAS CARACTERÍSTICAS PETROGRÁFICAS}

Para unas mismas condiciones de corte, no hay duda que la diferente cortabilidad de los distintos materiales rocosos hay que achacársela a sus diferentes características petrográficas (mineralogía, textura y espacios vacíos). Esto es lógico, ya que una roca está generalmente compuesta por una serie de minerales distintos (ocasionalmente el mismo, en las rocas monominerálicas, como las cuarcitas o los mármoles puros) que poseen diferente comportamiento mecánico, en forma de cristales de diferente tamaño colocados espacialmente según una disposición concreta (textura) y con unos espacios vacíos con formas planares (fisuras) o más equidimensionales (poros). Será, pues, el conjunto de todas estas características el que influya en su cortabilidad.

No obstante, éste es uno de los temas menos conocidos en el corte de materiales rocosos con discos diamantados, es decir, el conocimiento de la importancia que las características petrográficas de la roca cortada ejercen sobre el rendimiento del disco, a pesar de los intentos de diversos investigadores en descifrar esta influencia.

\subsection{La mineralogía}

Tradicionalmente es conocido que la proporción de cuarzo es uno de los factores petrográficos que influye en la cortabilidad de los materiales rocosos. Sin embargo, algunos investigadores indican que este parámetro aislado no puede utilizarse como indicador de la cortabilidad $(8,9$, $10)$.

No hay duda que la mineralogía ejerce una gran influencia sobre la cortabilidad y sobre el desgaste del disco: por ejemplo, la dureza Mohs (resistencia que ofrece un material a ser rayado) del cuarzo es 7 , mientras que el de la calcita es 3. Por tanto, el surco que el diamante deja en uno y otro mineral van a ser distintos para un mismo nivel de esfuerzo. Así, Bortolussi et al. (11) mencionan que en materiales frágiles (como los granitos) el surco producido inicialmente es neto, pero a medida que aumenta la carga, los bordes del surco aparecen pobremente definidos, debido a la saltación de material por las fisuras laterales. En los feldespatos (plagioclasas y feldespatos potásicos) el surco es más regular que en el cuarzo, y en las micas (biotita y moscovita) el surco es más profundo. En el caso de los granitos, el cuarzo es un mineral que es abundante (alrededor del 30-35 \%) en los "granitos s. str.", pero escaso o nulo en los "granitos negros" (como las anortositas y los gabros). En ambos tipos de granitos predominan los feldespatos (dureza Mohs: 6-6,5), pero más en los "granitos negros", por lo que éstos son más fáciles de cortar y menos abrasivos que los granitos s. str.. No obstante, en uno y otro caso no sólo hay que considerar la presencia de cuarzo, sino también la de los otros minerales a la hora de predecir su cortabilidad. Además, el grado de alteración o deterioro de los minerales (que cambia sus características elásticas) influye en la capacidad de arranque de material por parte de los diamantes (12).

\subsection{La textura}

La textura (que hace referencia a la forma, tamaño y disposición de los minerales en la roca) es otro factor que puede influir. El tamaño de grano ya ha sido considerado por Birle y Ratterman (13) y por Perrier (10), entre otros, como un parámetro que afecta a la abrasividad de la roca sobre el disco: a mayor tamaño de grano, mayor abrasividad. Del mismo modo, otras características texturales, como la orientación de los granos y la unión entre los cristales tienen una influencia sobre la predicción de la cortabilidad de un material (8). Cuando los cristales de diamante atraviesan a alta velocidad los contactos entre granos de diferente tenacidad, existe una alta probabilidad de que rompan, haciéndose los surcos más rugosos y a veces discontinuos (11).

\subsection{Los espacios vacíos}

La presencia de espacios vacíos también puede jugar un papel importante en el proceso de corte (14), contribuyendo a una cortabilidad mejor o peor: en general son más fáciles de aserrar los materiales mas porosos que los más compactos (15). Tanto los poros como las microfisuras contribuyen a reducir la unión textural de la roca, modificando sus características elásticas, y, por tanto, modificando los campos de esfuerzos creados durante el corte con disco. La zona de trituración y las fisuras medias, en cono y laterales producidas durante el corte interferirán con las fisuras o espacios vacíos de la roca facilitando el arranque de las partículas de roca y afectando, por tanto, a su cortabilidad.

En el caso de los granitos, la mayor parte de los espacios vacíos corresponden a morfologías de tipo fisura que, en muchas ocasiones, presentan orientaciones preferentes más o menos marcadas, confiriendo una cierta anisotropía a la roca. Esta orientación de las fisuras respecto a la dirección de corte de la roca también ejerce una influencia en la cortabilidad de la roca, por los motivos mencionados anteriormente. La forma del surco es más irregular cuando las microfisuras se encuentran dispuestas perpendicularmente a la dirección de corte (11).

Los intentos de encontrar una correlación entre la cortabilidad de las rocas y sus características petrográficas 
no han llegado a conclusiones generales, en nuestra opinión, debido a la falta de especialistas en Petrofísica (disciplina que interpreta las propiedades físicas de las rocas en función de sus características petrográficas) capaces de cuantificar dichos parámetros. Birle y Ratterman (13) ya indicaban las grandes dificultades que encontraban para cuantificar dichos parámetros así como los elevados costes que conlleva la obtención de estos datos. Sin embargo, hoy día, la utilización de la estereología y del proceso digital de imágenes aplicados a multiimágenes obtenidas mediante distintas técnicas microscópicas permitiría la cuantificación de los parámetros petrográficos de un modo más rápido y económico, sin excesivas dificultades $(16,17)$. Este tipo de estudios requiere una preparación especial de las muestras de roca (18).

\section{IMPORTANCIA DE LAS PROPIEDADES FÍSICAS}

Ante la falta de cuantificación de las características petrográficas, los autores que han investigado la influencia del tipo de roca sobre su cortabilidad o sobre la vida útil del disco han recurrido a caracterizar dichos materiales mediante el conocimiento de su composición mineralógica, fácil de obtener, y de algunas de sus propiedades físicas, especialmente aquéllas más directamente relacionadas con su comportamiento mecánico: resistencia a la compresión y tracción, dureza escleroscópica, resistencia a la abrasión, etc. $(13,19)$. Hay que tener en cuenta que la Petrofísica ha demostrado que todas estas propiedades físicas dependen de las características petrográficas de los materiales, por lo que éste sería un modo de suplir la dificultad que encierra la cuantificación de estos parámetros petrográficos.
Los estudios realizados por diversos autores $(8,9,13)$ establecen la dificultad de correlacionar estas propiedades físicas con la cortabilidad del material. Por ejemplo, los datos obtenidos por Burgess y Birle (8) y Birle y Ratterman (13) respecto a la cortabilidad de 10 rocas graníticas no se correlacionan con ninguna de las tres propiedades físicas de los granitos que ellos consideraban más importantes (contenido en cuarzo, resistencia a la abrasión y dureza escleroscópica) (Fig. 2 a, b y c), lo mismo que la abrasividad tampoco depende exclusivamente del contenido en cuarzo (Fig. 2 d). No obstante, Burgess y Birle (8) llegaron a una aproximación respecto a la cortabilidad (expresada como la superficie cortada por milímetro de desgaste del diámetro del disco, $\mathrm{m}^{2} / \mathrm{mm}$ ) y algunas características petrográficas y propiedades físicas de los materiales cortados, de modo que:

$$
\mathrm{Y}=27,77-0,16 \mathrm{~B}-0,12 \mathrm{C}-0,10 \mathrm{D}
$$

siendo
Y: Cortabilidad $\left(\mathrm{m}^{2} / \mathrm{mm}\right)$
B: Resistencia a la abrasión
C: Dureza escleroscópica (Dureza Shore)
D: Porcentaje volumétrico de cuarzo

Los autores recalcan que esta ecuación sólo es válida para las condiciones específicas de realización del ensayo y que, en otros casos, habría que determinar otra ecuación.

Posteriormente, y como continuación de estos estudios, Birle y Ratterman (13) consideran, además de las tres características anteriores, el porcentaje de feldespato potásico, plagioclasas y otros minerales así como el tamaño medio de grano del cuarzo.

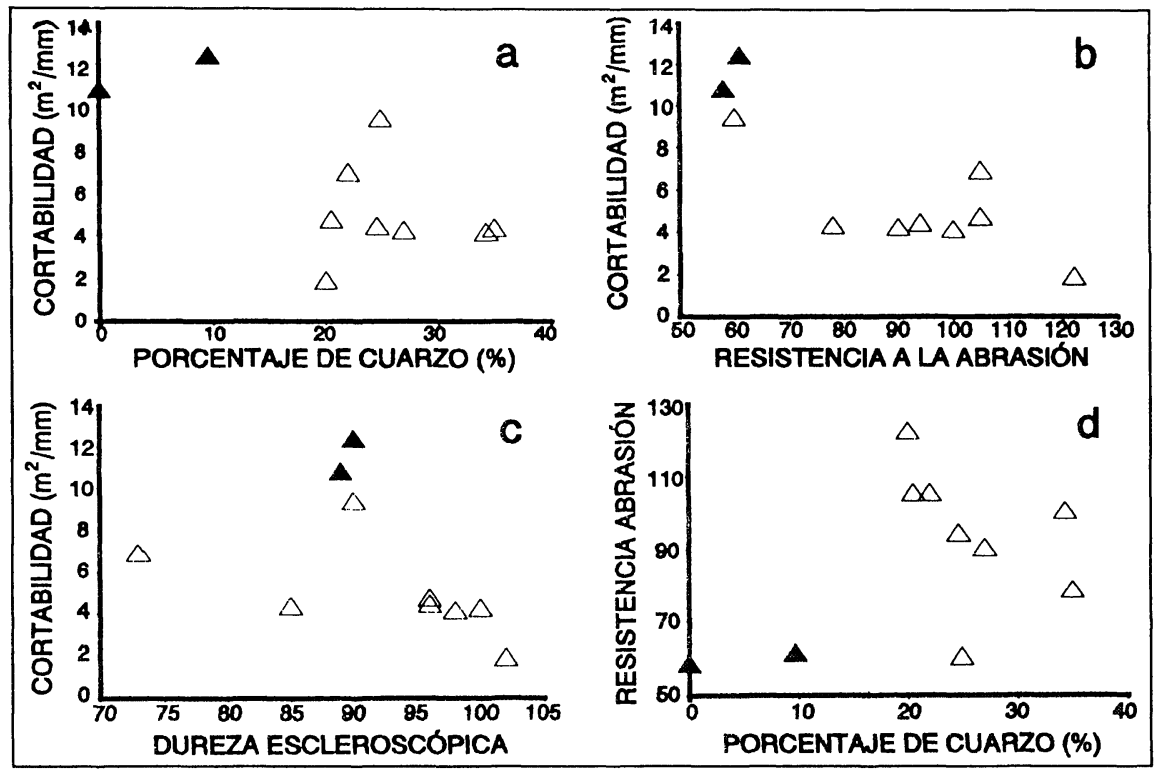

Fig. 2.- Relación entre la cortabilidad y algunos parámetros petrográficos y físicos de algunos granitos (los puntos negros corresponden a dioritas). (A partir de los datos de Birle y Ratterman (13)). 
De todas estas variables sólo dos resultaron significativas $(F>0,15)$ en la regresión: la resistencia a la abrasión según norma ASTM C 241-51 (B) y el porcentaje de cuarzo (D) de modo que:

$$
Y=18-0,13 D-0,10 B
$$

La dureza escleroscópica que en la ecuación de Burgess y Birle (8) tenía mayor influencia que el porcentaje de cuarzo, ya no aparece. Además, en esta segunda ecuación, el porcentaje de cuarzo tiene una mayor influencia que la resistencia a la abrasión. No obstante, ambas ecuaciones (válidas para unas condiciones específicas, como ya se ha mencionado) hay que considerarlas con cierta precaución ya que los autores mencionan que los análisis petrográficos se llevaron a cabo sobre un número pequeño de láminas delgadas ( 1 ó 2 por cada material), lo que puede inducir a unos errores de cálculo muy importantes y que dependen, fundamentalmente, del tamaño de grano de los materiales.

Con esta ecuación calcularon la cortabilidad de 52 rocas comerciales, clasificándolas en 4 grupos:

Grupo I Cortabilidad $>7 \mathrm{~m}^{2} / \mathrm{mm}$

Grupo II Cortabilidad entre 7 y $4 \mathrm{~m}^{2} / \mathrm{mm}$

Grupo III Cortabilidad entre 4 y $3 \mathrm{~m}^{2} / \mathrm{mm}$

Grupo IV Cortabilidad $<3 \mathrm{~m}^{2} / \mathrm{mm}$

Perrier (10) completa los datos de Birle y Ratterman (13) citando las recomendaciones de corte de General Electric para las cuatro categorías de granitos en lo que se refiere a la profundidad de corte y al régimen de corte.

Wright y Cassapi (9) también intentaron establecer la cortabilidad de algunas rocas en función de una serie de características petrográficas y propiedades físicas:

- Composición mineralógica (cuarzo, feldespato potásico, plagioclasa y otros)

- Tamaño y forma de los cristales

- Dureza Mohs media de cada roca

- Dureza escleroscópica Shore

- Indentación del cono NCB (National Coal Board, UK)

- Abrasividad Cerchar

- Resistencia a la compresión

- Resistencia a la tracción indirecta (ensayo brasileño).

Los resultados no fueron muy alentadores, llegando a la conclusión que no existe una relación sencilla entra la cortabilidad y las características petrográficas y físicas de los materiales, ya que en el proceso de corte intervienen muchos factores. No obstante, estos autores proponen utilizar la medida de las fuerzas que se generan en el corte para estimar la cortabilidad. Para ello utilizaron una mesa de corte que permitía medir tanto la fuerza vertical como la horizontal mediante una serie de transductores de fuerza. De hecho, encontraron una buena correlación entre las fuerzas verticales y el desgaste del disco, que permitía utilizar estos valores para estimar la cortabilidad del material.

A pesar de la dificultad que entrañaba la interpretación de la cortabilidad, estos autores afirman que la información obtenida en los estudios petrográficos puede ser muy ventajosa para determinar las especificaciones de los útiles de corte.

Bortolussi et al. (14) afirman, si bien se refiere al corte con hilo diamantado, que el parámetro que reviste una mayor importancia para caracterizar la cortabilidad del material es la resistencia a la abrasión, mientras que otras propiedades como la resistencia al impacto, a la compresión uniaxial y a la tracción parecen tener poca influencia. Además, el módulo de elasticidad y el estado de microfisuración pueden jugar un papel importante.

Con el fin de dar una orientación respecto a las condiciones de corte (velocidad de corte, velocidad periférica del disco, profundidad de corte, etc.) e incluso de las características del disco (tamaño, tipo y concentración del diamante), se han establecido algunas clasificaciones de indole práctico. Entre ellas se puede mencionar la propuesta por la firma Winter \& Sohn que divide a las rocas en 6 categorías ( 0, I, II, III, IV y V) de acuerdo con la producción media $\left(\mathrm{m}^{2} / \mathrm{h}\right)$ de las mismas. Por ejemplo, la clase 0 comprende las rocas calcáreas; la clase I, los granitos negros y el Labrador Oscuro; a la clase II pertenece el Labrador Claro y a la clase III, el Granito de Tarn; la clase IV incluye al Rojo Balmoral, el Marrón Báltico, el Halmstad y el Vanga y la clase $\mathrm{V}$ comprende a rocas como el Rojo de Assuan, el Rojo de Sardinia, al Rojo de 'Tranas y la Cuarcita de Wasa.

La compañía General Electric, basándose en los cuatro grupos de rocas establecidos por Birle y Ratterman (13) comentados anteriormente, establece los tipos de diamante que deben ser utilizados según el tipo de trabajo a realizar. Por su parte, la compañía Diamant Boart establece 5 clases de granitos según la producción media: la clase $\mathrm{I}$, entre 3 y $2,4 \mathrm{~m}^{2} / \mathrm{h}$; la clase II, entre 2,4 y $1,8 \mathrm{~m}^{2} / \mathrm{h}$; la clase III, entre 1,8 y $1,2 \mathrm{~m}^{2} / \mathrm{h}$; la clase IV, entre 1,2 y $0,9 \mathrm{~m}^{2} / \mathrm{h}$, y la clase $\mathrm{V}$, entre 0,9 y $0,6 \mathrm{~m}^{2} / \mathrm{h}$. Además recomienda la velocidad periférica del disco (de 38 a $23 \mathrm{~m} / \mathrm{s}$ ) y la profundidad de corte (de 20 a $4 \mathrm{~mm}$ ) para cada clase de roca (Figura 3).

Finalmente, también otros organismos han establecido sus clasificaciones de rocas según su cortabilidad. Así, en Francia, el Syndicat des Fabricants d'Outillage des Produits Base de Diamant (SINFODIA) estableció 5 clases de acuerdo con la abrasividad de la roca al disco y al comportamiento del corte, recomendando algunos parámetros específicos del corte para cada clase 


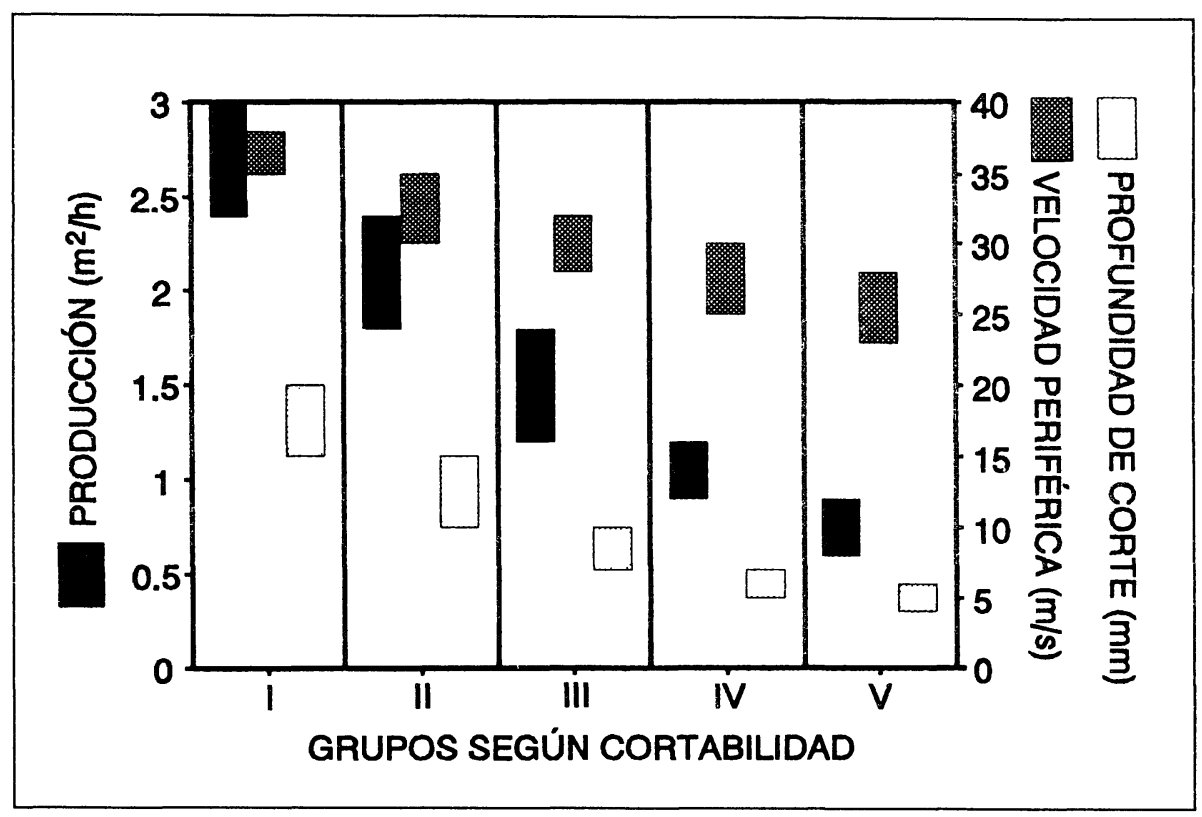

Fig. 3.-Clasificación de las rocas según su cortabilidad y recomendaciones de corte con discos diamantados de Diamant Boart (en 10).

(especialmente profundidad de corte y velocidad transversal), aunque según Perrier (10) esta clasificación ya no está en uso. En Alemania, el Fachverband Werkzeugindustrie e.V. (FWI) propone tres categorías de roca dura, recomendando también determinadas velocidades de corte.

\section{CONCLUSIONES}

El rendimiento de los útiles diamantados, especialmente los discos, en el corte de rocas ornamentales está influenciado por una serie de características propias del proceso de corte (tipo y tamaño de disco, potencia de la sierra, velocidad de corte, etc.) y otras propias de la roca en sí (mineralogía, textura y espacios vacíos). En general, los estudios realizados han hecho poco hincapié en las características petrográficas de los materiales a cortar para predecir su cortabilidad. El desarrollo de las técnicas de análisis de imágenes permite hoy día cuantificar los parámetros petrográficos de un modo menos costoso y mucho más rápido que hace algunos años, por lo que, en nuestra opinión, deberían involucrarse los investigadores en un ambicioso programa de caracterización petrofísica de rocas ornamentales para llegar a conclusiones objetivas respecto a su cortabilidad. Esto permitiría clasificar las rocas previamente a su corte y seleccionar las condiciones de corte más idóneas.

Por nuestra parte, hemos comenzado a caracterizar diversos granitos españoles desde el punto de vista petrográfico para intentar correlacionar sus características con la velocidad de corte, cuando éste se realiza en condiciones de presión constante. A la espera todavía de datos definitivos y concluyentes, los resultados preliminares parecen prometedores.

\section{AGRADECIMIENTOS}

A la Universidad de Oviedo, por la subvención SV-VIC95-0027 concedida para la realización de este trabajo.

\section{BIBLIOGRAFÍA}

(1) SUÁREZ DEL RÍO, L.M.; A. RODRÍGUEZ REY, L. CALLEJA Y V.G. RUIZ DE ARGANDOÑA (1968). "El corte de rocas ornamentales con discos diamantados: influencia de los factores propios del sistema de corte". Materiales de Construcción, Vol. $48, \mathrm{n}^{\circ} 250$, pp. 53-59, abril/junio 1998.

(2) HOWARTH, D.F.; W.R. ADAMSON Y J.R. BERNDT (1986). "Correlation of model tunnel boring and drilling machine performances with rock properties”. Int. J. Rock Mech. Min. Sci. \& Geomech. Abstr. Vol. 23, n² 2, pp. 171-175.

(3) SWAIN, M.V. Y B.R. LAWN (1976). "Indentation fracture in brittle rocks and glasses". Int. J. Rock Mech. Min. Sci. \& Geomech. Abstr., 13, pp. 311-319. 
(4) WILKS, J. Y E. WILKS (1991) "Properties and application of diamond”. 1 ${ }^{\text {a }}$ Edición. Ed. Butterworth-Heinemann, Oxford. $525 \mathrm{pp}$.

(5) McGOWAN, J. Y G. BRANNINGER (1991) “The application of superabrasives in granite slabbing”. Superabrasives'91 . junio, 11-13, 1991. Chicago, Illinois. (Existe una traducción al español de este trabajo en Roc Máquina, nov. 91, pp. 97-110).

(6) GENERAL ELECTRIC (1989) "Future diamond bright for granite building materials". Dimensional Stone, enero/febrero 1989.

(7) BRAUNINGER, G. Y J. McGOWAN (1991) "La aplicación de los superabrasivos en el corte de placas de granito". Roc Máquina, noviembre 1991, pp. 97-110.

(8) BURGESS, R.R. Y J.D. BIRLE (1978) “El corte de granito con discos de diamante". 30 Asamblea Seminario de la Asociación de Diamante Industrial de Japón. Tokyo. (Traducido al castellano por General Electric). También ha aparecido en Roc Máquina, 3er trimestre de 1989, pp. 22-35.

(9) WRIGHT, D.N. Y V.B. CASSAPI (1985) "Factors influencing stone sawability". Industrial Diamond Review, 2/85, pp. 8487.

(10) PERRIER, R (1993) “Le diamant dans le sciage circulaire des roches. 3ème partie”. Le Mausolée, 5/93, pp. 44-49.

(11) BORTOLUSSI, A.; A. CARANASSIOS; R. CICCU; R. LASSANDRO; P.P. MANCA Y G. MASSACCI (1992) "Progress in the knowledge of granite cutting with diamond wire". 11 th Int. Conf. on Ground Control in Mining, The University of Wollongong, N.S.W., pp. 593-599.

(12) CALLEJA, L.; V.G. RUIZ DE ARGANDOÑA; C. DE LLANOS; L.M. SUÁREZ DEL RÍO Y A. RODRÍGUEZ REY (1995). "Estudio de la deformación producida por cortes de precisión en rocas graníticas". Comunicaciones de la XVII Reunión Bienal de la Sociedad Española de Microscopía Electrónica. Oviedo, abril 1995. pp. 284-285.

(13) BIRLE, J.D. Y E. RATTERMAN (1986) "Resumen sobre la capacidad de corte de la roca dura basado en pruebas de laboratorio". General Electric (traducción de una publicación de Dimensional Stone Magazine).

(14) BORTOLUSSI, A.; R. CICCU; P.P. MANCA Y G. MASSACCI (1990) "Simulation and optimization of rock cutting with diamond wire". Proc. APCOM: 1990; 22nd Int. Symp., 17-21 sept. 1990, Berlín, pp. 164-176.

(15) PERFETTI, F.; P. MANNELLA Y P. GIANNINI (1993) "La técnica del aserrado de granito con granalla (II)". Roc Máquina, febrero 1993, pp. 73-94.

(16) MONTOTO, M.; A. RODRÍGUEZ-REY; B. MENÉNDEZ; A. MARTÍNEZ NISTAL; V.G. RUIZ DE ARGANDOÑA; L.M. SUÁREZ DEL RÍO Y L. CALLEJA (1993) “Microfractography of "El Berrocal” granite”. Proc. 5th NAWG \& ARAP (Toledo), pp. 303-308.

(17) ÁlVAREZ CAlleJA, A.; A. RODRÍGUEZ-REY; M. MONTOTO; B. MENÉNDEZ Y C. DUBOIS (1993) "Cuantificación mediante estudio estereológico del sistema microfisural del granito de El Berrocal (Sistema Central, Toledo, España)". Acta Geológica Hispánica, Vol. 28, $\mathrm{n}^{\circ} 4$, pp. 63-69.

(18) MONTOTO, M.; A. RODRÍGUEZ REY; L.M. SUÁREZ DEL RÍO; L. CALLEJA Y V.G. RUIZ DE ARGANDOÑA (1987) "Methodology for microscopic studies of alteration of Building stones". Sixth Meeting of the European Clay Groups, Euroclay' 87 (Round Table on: The Decay of Building Stone) E. Galán y M.A. Vázquez (Eds.) pp. 45-48.

(19) JENNINGS, M. Y D. WRIGHT (1989) “Guidelines for sawing stone”. Industrial Diamond Review, 2/89, pp. 70-75. 